\title{
CHOREA AS THE FIRST MANIFESTATION OF SYSTEMIC LUPUS ERYTHEMATOSUS: A CASE REPORT
}

Rebecca de Souza Costa ${ }^{1, \star}$, Dalila lari Silva Barbosa ${ }^{1}$, Thais Chab Campano Latorre ${ }^{1}$, Cassiano Henrique Geovanini Vanzeli ${ }^{1}$, Bruno de Mendonça Moraes Araújoํㅗㄹ José Eduardo Martinez ${ }^{1}$

1.Pontifícia Universidade Católica de São Paulo, São Paulo (SP), Brazil.

*Corresponding author: rebecca.s.costa@hotmail.com

\section{BACKGROUND}

Systemic lupus erythematosus (SLE) is a disorder of the immune system with a wide range of symptoms. The initial condition usually presents with constitutional symptoms, which may be accompanied by arthralgias or arthritis. The involvement of kidneys, lungs and central nervous system (CNS) denotes a more severe character and worse prognosis. Several neurological and psychiatric manifestations may be present, the most common being convulsion, neuropathies and acute confusional state, but chorea is seen in less than $2 \%$ of patients. Next, we will bring the case report of a patient whose first manifestation of SLE was chorea associated with the skin condition.

\section{CASE REPORT}

ZCRG, female, 22 years old, referred to the CHS for presenting chorea movements and malar rash. She reports that six months ago she started a picture of uncoordinated movements in her right upper limb and malar rash associated with photosensitivity. He looked for UBS and was prescribed clonazepam. Patient progressed progressively with worsening of movements, generalizing to upper and lower limbs, typical of chorea. On physical examination, the patient was discolored +/4+, skin: malar rash, neurological: slurred speech, uncoordinated movements, conscious and oriented. Other systems without changes. Laboratory tests on admission: CBC and renal function without changes, PCR: $54.9,24 \mathrm{~h}$ proteinuria: $600 \mathrm{mg}$, negative serology for viral hepatitis, HIV and VRDL. A 1:320 fine speckled nuclear standard ANA. Diagnostic hypothesis: SLE neurological and skin activity. Haloperidol $1 \mathrm{mg} / \mathrm{day}$, hydroxychloroquine $400 \mathrm{mg} /$ day and prednisone $60 \mathrm{mg} /$ day were started. The patient evolved with an improvement in the initial clinical condition, being discharged from the hospital. Returns to the rheumatology clinic in two months, no longer presenting malar rash and chorea, with a reduced dose of corticosteroids.

\section{CONCLUSION}

The involvement of SLE in the CNS still represents a great challenge in clinical practice and its pathophysiology is not completely known. Differential diagnoses should always be considered in these cases: infectious, degenerative, metabolic disorders or medication use. Some studies show that these syndromes may be associated with the presence of antiphospholipid antibodies and that thrombotic events would be related to their pathophysiology. Chorea is a rare event and mainly affects women in their teens. The causes of these events are the result of microvasculopathy, thrombosis, presence of autoantibodies or the involvement of inflammatory mediators, and the proposed treatment is based on the use of corticosteroids and symptomatic therapy with dopamine antagonists.

\section{KEYWORDS}

Systemic lupus erythematosus, Chorea, Dopamine antagonists. 